\title{
La comunicación financiera en tiempos de crisis: el caso de los "bancos malos"
}

\author{
$\mathrm{M}^{\mathrm{a}}$ del Carmen de la ORden DE LA CRUZ \\ Universidad Rey Juan Carlos de Madrid \\ carmen.delaorden@urjc.es
}

\begin{abstract}
Resumen:
La crisis ha tenido un coste muy fuerte para una ciudadanía que no comprende sus orígenes ni soluciones por su escasa cultura financiera. No encuentra fuentes fiables y comprensibles dónde aprender y para informarse tiene que acudir a medios especializados que utilizan una jerga técnica complicada. El caso del denominado "banco malo" es un ejemplo; una acepción que, a priori, tiene un sentido negativo, cuando en realidad es una solución. El presente trabajo pretende hacer un acercamiento general a los "bancos malos" susceptibles de ser conocidos por la ciudadanía en tiempos de crisis.
\end{abstract}

Palabras clave:"Banco malo", comunicación a la ciudadanía, crisis bancarias, riesgo sistémico

\section{The financial communication in times of crisis: the case of "bad banks".}

\begin{abstract}
:
The crisis has had a very strong cost for citizens who do not understand their origins or solutions by low financial literacy. There are not reliable and comprehensive sources where they learn and if they want to learn more broadly, they have to go to specialized media using complicated technical jargon. The case of the so-called "bad bank" is an example of this situation, a sense that, a priori, has a negative meaning, when in fact it is a positive response to a problem in the banking sector. This paper addresses this solution that the citizen should know.
\end{abstract}

Key Words:Bad bank, communication to the citizens, banking crisis, financial culture, systemic risk

\section{Referencia normalizada:}

De la Orden de la Cruz, M. C. (2013) La comunicación financiera en tiempos de crisis: el caso de los "bancos malos". Historia y Comunicación Social. Vol. 18 N Especial Octubre. Págs. 813-822.

Sumario: 1. Introducción y metodología. 2. El "banco malo" como solución. 3. El concepto de "banco malo". 4. El diseño del "banco malo" y gestión de los activos problemáticos. 5. Conclusiones. 6. Referencias bibliográficas. 


\section{Introducción y metodología}

El coste de la crisis financiera ha sido amplio y ha llegado a todas las capas de una ciudadanía con una escasa cultura financiera, que no sabe lo suficiente y que no encuentra fuentes fiables y comprensibles dónde aprender. Cuando quiere ampliar la información, tiene que acudir a medios especializados que utilizan una jerga técnica, a veces complicada.

Un ejemplo de esta situación es la solución que las autoridades financieras han dado a la crisis del sector bancario en Europa con la creación de los denominados "banco malos". Un nombre que, a priori, señala un sentido negativo, cuando en realidad es una respuesta positiva a un problema de carácter financiero. Saber qué es un "banco malo" es imprescindible para transmitir tranquilidad a los ciudadanos, elemento que se configura como uno de los apoyos fundamentales en la salida de cualquier crisis. El presente trabajo pretende hacer un acercamiento general a los bancos malos y a todas sus características, susceptibles de ser conocidas por la ciudadanía en tiempos de crisis.

El estudio de las causas y cómo resolver los desequilibrios generados por una crisis constituyen temas permanentes de estudio (entre otros, Acharya y Mattew 2009; BIS, 2008 y 2009; Borio y Nelson, 2008; Calomiris, 2008; Diamond y Rajan, 2009; Eichengreen et al., 2009; Harvey, 2008; IMF, 2008; Praet y Nguyen, 2008; Swagel, 2009; y Taylor, 2009). Uno de los elementos de esta crisis proviene de los excesos cometidos en las épocas de bonanza, en las que se produce una expansión crediticia que lleva a grandes desequilibrios en los balances del sector privado y que finalmente, se trasladan a un riesgo de crédito en el sector bancario. Las instituciones financieras, generalmente, infravaloran los riesgos de su actividad y terminan en crisis bancarias. Según Laeven y Valencia (2010), un tercio de estas crisis ha venido precedido por un boom crediticio y normalmente, las crisis cambiarias y de deuda tienden a producirse después. Las crisis bancarias se producen de forma simultánea con otras, más con las cambiarias que con las de deuda $y$, en menor medida, se originan las tres al mismo tiempo.

Las crisis bancarias que se han producido desde 2007 presentan características similares a las registradas anteriormente, pero también diferencias (Laeven, 2010). En esta última ocasión, la crisis se ha focalizado en los países desarrollados con un alto grado de conexión internacional. La velocidad de la intervención y la implantación de medidas, tanto de política económica como otras de carácter inusual, ha sido más rápida y más amplia; y los costes han sido mayores en términos económicos y de aumento de endeudamiento público. Este último elemento ha generado preocupación sobre la sostenibilidad fiscal. De hecho, los costes de una crisis bancaria son mayores y más dañinas para la economía cuanto mayor grado de bancarización presenta una economía (Kroszner et al., 2007). A ello, se ha sumado la complejidad de los activos, especialmente los productos estructurados de crédito que han supuesto un elemento adicional por la dificultad para determinar su valoración y su papel como instrumentos de transferencia del riesgo de crédito (Borio et al. 2010). 
El método utilizado en este trabajo se ha basado en una investigación longitudinal y cualitativa sobre los principales autores que han estudiado y analizado las crisis bancarias y sus soluciones. El objetivo es conocer los elementos de una crisis bancaria y los mecanismos de resolución y saneamiento del sector. En concreto, el caso de los "bancos malos" analizando sus características, diseño y gestión de los "activos malos" que le han sido transferidos. En las siguientes líneas se aborda los diferentes aspectos de este tipo de sociedad para que la ciudadanía pueda conocerlos, sin quedarse únicamente, en su apelativo temido y peyorativo.

\section{El banco malo como solución}

La forma de afrontar las crisis bancarias y las soluciones han sido ampliamente estudiadas y han ido cambiando con el paso del tiempo, no habiendo una única u óptima solución (Klingebiel, 2000), especialmente cuando llega a ser una crisis sistémica. Los principios generalmente aceptados como básicos para una gestión adecuada de una crisis bancaria son: la velocidad en el reconocimiento de su existencia y en la intervención, la fuerza y profundidad de la respuesta y cómo equilibrar los costes con la contención del riesgo moral (Borio et al., 2010). En este sentido, Laeven y Valencia (2012) señalan que las actuaciones de política económica son más intensas en los países desarrollados que en los emergentes. Esto puede llevar a actitudes de los agentes económicos más arriesgadas apareciendo el denominado riesgo moral. Otro factor que influye en la forma de actuar ante una crisis es la estructura institucional y política de cada país y sus relaciones. Los países que presentan una organización con capacidad de negociación colectiva están más preparados para diseñar un plan de rescate nacional más equilibrado y adecuado (Grossman y Woll, 2012). En concreto, las medidas que han adoptado los Estados para afrontar una crisis bancaria, tradicionalmente, se han articulado a través de soluciones que afectan al pasivo de los balances de los bancos: inyecciones directas de capital a los bancos (parciales o totales -nacionalizaciones-) o dando garantías explícitas para seguir teniendo acceso a la financiación; y a través del activo, comprando o garantizando los activos problemáticos para reducir el riesgo de pérdida de los bancos, haciéndolos viables (Panetta et al., 2009; Laeven y Valencia, 2008).

Decidida esta última opción, las posibilidades de gestión de los activos problemáticos son tres: la garantía de los mismos, la segregación o un mecanismo mixto. En el aseguramiento de activos, los bancos mantienen los activos problemáticos, pero reciben una ayuda del Estado para compensar las pérdidas. Esta alternativa presenta problemas importantes como las dificultades en el cálculo de la prima y su aplicación porque suelen estar repartidos entre muchos bancos y se pueden generar conflictos internos de intereses por la complejidad de separar claramente los activos problemáticos de los sanos. En cuanto a la segregación de activos problemáticos de los sanos, ésta se puede hacer a través de la compra directa y la creación de una o varias socie- 
dades con algún tipo de garantía pública o propiedad estatal, son los denominados "bancos malos".

Este tipo de instituciones ha sido utilizado como mecanismo de saneamiento del sector bancario desde los años 70 del siglo pasado y su popularización se produjo tras la crisis asiática del año 1997 (Claessens et al., 1999). De acuerdo con Laeven (2008), de las 42 crisis bancarias sistémicas analizadas entre 1970 y 2007, en 37 países, en el $48 \%$ de los casos se crearon agencias especiales para reestructurar bancos con problemas y en el $60 \%$ se estableció un "banco malo" para gestionar los activos problemáticos. En el pasado, las experiencias más significativas fueron en los años 80 en Estados Unidos con la creación de la sociedad RTC (Resolution Trust Corporation) para resolver los problemas generados por el excesivo riesgo asumido por las cajas de ahorro y, en los 90, Suecia estableció dos sociedades (Securum y Retriva) para afrontar los problemas financieros de los principales bancos suecos afectados por la burbuja inmobiliaria. Ahora, tras la crisis financiera iniciada en 2007, se han creado más de 15 "bancos malos" con diferentes formatos. El volumen de activos problemáticos traspasados a bancos malos en la crisis 2008-2009, osciló entre los 30 y 100 mil millones de euros, según el país considerado (Aggarwal, 2012).

\section{El concepto de "banco malo"}

Realmente, este tipo de sociedades siempre ha existido en la actividad bancaria habitual, como unidades internas o entidades subsidiarias externas para dar solución a los activos problemáticos del banco. Pero la acepción de "banco malo" se utiliza como tal cuando se crean una o varias sociedades a gran escala y a nivel nacional, con la intervención del Estado y debido al elevado volumen de activos problemáticos de los bancos; lo que conlleva un riesgo sistémico. Las ventajas de establecer un "banco malo" son mayores si el volumen de activos es elevado y se dispone de bastante tiempo para liquidarlos (Bergstrom, 2003).

Centrándonos en su definición, el denominado "banco malo" (en inglés, "asset management company" -AMC- o "bad bank") es un acuerdo institucional, generalmente dirigido por el Estado, para crear un vehículo, bajo un formato de sociedad o fondo de carácter público y/o privado, que resuelva los activos problemáticos existentes en entidades de crédito de un sistema financiero con riesgo de generar una crisis bancaria sistémica. Constituye un mecanismo de respuesta cuando el volumen de estos activos en el sistema financiero es tan alto que crea incertidumbre sobre la solvencia de una o varias entidades financieras, generando desconfianza finalmente en todo el sector. La creación de un "banco malo" permite separar los activos "buenos" de los "malos", consiguiendo el saneamiento de los balances de las entidades financieras. Esto reduce la incertidumbre sobre su viabilidad. De hecho, evita el "juego de resurrección", un comportamiento al que tienden los directivos de entidades excesivamente endeudadas, al gestionar de una forma más arriesgada en un intento desespe- 
rado de salvarlas (Freixas et al., 2003). Con el saneamiento del balance, los directivos tienen la oportunidad de reflotar la entidad sin riesgos añadidos.

Los activos problemáticos, también denominados "tóxicos" o "dañados", son aquéllos que presentan un alto riesgo de crédito o están sometidos a bruscos ajustes de valoración y, generalmente, son ilíquidos en su mercado (Ayuso y del Río, 2012). Los activos problemáticos tradicionales han sido las inversiones crediticias y los activos reales derivados de la ejecución de préstamos con garantía, generalmente inmuebles. Pero en la crisis financiera iniciada en 2007, la gama de activos se ha ampliado a otros más complejos, catalogándolos la Comisión Europea (CE) como "cestas", en los que se incluyen los denominados productos de financiación estructurada y titulizados (RMBS -Residential Mortgage-Backed Security-, CMBS -Commercial Mortgage-Backed Security-, CDO -Collateralized Debt Obligation-, ABS -Asset-Backed Security-). También contempló como tales otros activos con causas debidamente justificadas, como el caso de los activos inmobiliarios procedentes del estallido de la burbuja inmobiliaria. Incluso, se dio la posibilidad de considerar activos adicionales sin necesidad de justificación específica. El objetivo era considerar una gama de activos lo suficientemente amplia para hacer un programa de rescate completo (CE, 2009). Los tipos de activos traspasados condicionan la gestión del "banco malo". Klingebiel (2000) señala el grado de liquidez de estos activos como uno de los factores clave para el éxito de la gestión de los "bancos malos". Así, se hace necesario realizar una clasificación de activos en función de una serie de variables estandarizadas para establecer una prioridad de resolución (Aggarwal et al., 2012).

De este modo, el objetivo de la creación de los bancos malos es doble. Uno, de carácter específico, eliminando el riesgo de los activos problemáticos en las entidades financieras consiguiendo hacerlas viables y otro, de carácter amplio, al reducir el riesgo para todo el sistema en su conjunto con el menor coste posible. En concreto, para el propio sistema bancario, evitando la quiebra de entidades, mejorando su solvencia, restaurando la actividad del mercado del crédito y por ende, la confianza en el sector financiero.

\section{El diseño de los "bancos malos" y la gestión de "activos malos"}

Cómo definir la estructura más adecuada con uno o varios bancos malos es compleja y depende de muchos factores. No existe un sólo modelo de "banco malo" ya que hay varias formas de gestionar los activos problemáticos. Esto ha generado el clásico debate entre el modelo centralizado o descentralizado (Klingebiel, 2000). Cuando se trata de una gestión totalmente centralizada, se crea una sola entidad para todo el sistema financiero en su conjunto con el objetivo de guiar el proceso de reestructuración de forma global (García, 1999; Claessens et al., 1999). Esta variante puede conllevar una operación previa de nacionalización de los bancos con problemas o incluso, de todo el sector bancario (CE, 2009) y se hace una reestructuración 
conjunta del sector. Esta alternativa genera importantes ventajas al conseguir importantes economías de escala en diversos aspectos de la gestión, pero también aparecen problemas derivados de su gran tamaño y estructura del accionariado, pudiéndose producir presiones políticas. La alternativa de una gestión centralizada ganó especial importancia a partir de la crisis asiática de 1997 (Klingebiel, 2000). Laeven y Valencia (2008) confirman que, generalmente, se opta por ella, creándose este tipo de entidades en los momentos críticos de la crisis cuando se alcanza el máximo de préstamos morosos. En el caso de ser descentralizado, se establecen varios bancos malos, uno por cada entidad involucrada, siendo entidades subsidiarias privadas del banco bien capitalizadas. La principal ventaja es su especialización, pero al ser puede conllevar un riesgo de maquillaje de cifras al ser gestionada por la entidad original.

Elegido un modelo $u$ otro, se ha de definir un adecuado plan de negocio que convenza a todos los agentes implicados y genere la suficiente confianza. Uno de los aspectos más importantes son las fuentes de financiación y el tipo de los inversores objetivo. Estas sociedades están financiadas principalmente con fondos públicos. Generalmente, cuando asume activos muy problemáticos es difícil que se consiga financiación del sector privado, salvo que se establezcan garantías por parte del Estado del valor futuro de la cartera de los activos, algo que es complejo de ofrecer (Schäfer y Zimmermann, 2009). La alternativa es que el Estado asuma totalmente el riesgo y consiga realizar una liquidación gradual intentando preservar el valor de los activos. Por ello, también es importante que el plazo de vida que se establezca para el "banco malo" sea lo suficientemente amplio.

La valoración de los activos problemáticos en el momento del traspaso constituye otro elemento importante. La CE (2009) estableció una metodología general reconocida internacionalmente como es el denominado "valor económico real" establecido según cestas de activos y bajo unas hipótesis prudentes y realistas, con reevaluación periódica. En el caso de activos de valoración compleja, se contemplaban aplicar descuentos uniformes sobre el valor por tipo de cestas. Una vez determinado, el valor de traspaso tenía que situarse por debajo del económico real para que el Estado fuera compensado por los riesgos asumidos (Schäfer y Zimmermann, 2009).

Una vez transferidos los activos, el precio de liquidación sigue siendo una cuestión importante presentando el problema de valoración al que se añade el ritmo de ventas como factor de discusión pues no hay una postura única. Una venta rápida puede establecer un suelo de precios que finalmente favorezca las compras (CE, 2009). En un proceso de venta lenta, el "banco malo" puede ir vendiendo a mejores precios bajo un escenario de recuperación económica. Sin embargo, retrasar las ventas de los activos problemáticos esperando a que recuperen valor supone asumir el riesgo de una caída fuerte de los precios, ajustándose la valoración en la oferta futura de activos (Shleifer y Vishny, 1992; Lang et al., 1995). En este sentido, el establecimiento de un horizonte de tiempo lo suficientemente largo, para liquidar los activos sin presiones hasta el final, juega un papel importante. Habitualmente, éste se ha situado entre los 10 y 15 años. 
La capacidad para poder vender a un mayor o menor ritmo depende también del margen de maniobra financiero que tenga el Estado. Si está poco endeudado y puede soportar los costes, la venta se hace más pausada. En caso contrario, conviene un ritmo rápido (Aggarwal, 2012). También hay que considerar el volumen de activos a liquidar. Bergstrom et al. (2003) defienden que una venta rápida sólo se puede llevar a cabo cuando hay un pequeño volumen de activos para dar salida. En caso contrario, se necesita más tiempo para evitar el hundimiento de los precios. Pero si hay un stock muy grande, el precio tarda mucho tiempo en recuperarse por el exceso de oferta. De este modo, no hay una evidencia concluyente sobre el ritmo de ventas. Una alternativa a este inconveniente es el establecimiento de incentivos, incluso negativos, o alternativas como la refinanciación que faciliten la venta o liquidación. Ergungor (2007) pone como ejemplo el del gobierno mexicano después de la crisis del Tequila, con subsidios a los morosos ligados al pago de los créditos. En todo caso, se hace necesario definir una estrategia de ventas intentando evaluar cuál puede ser el ritmo de salidas, identificando potenciales compradores y evitando dar una imagen de "vendedor forzado" (Aggarwal et al., 2012).

Otro elemento clave para el éxito de un "banco malo" es el establecimiento de un gobierno corporativo adecuado y una gestión profesionalizada, libre de interferencias políticas (Schäfer y Zimmermann, 2009; Aggarwal et al., 2012). La gestión debe ser activa e implica negociaciones con deudores, reestructurar deudas y, si es necesario, condonar deuda. En este sentido, tener bien identificado y conocer al deudor para la negociación es muy importante (Schäfer y Zimmermann, 2009). Si bien, en muchas ocasiones, se requiere de equipos nuevos para dar una solución más rápida (Aggarwal et al., 2012). Y los incentivos de compensación para los gerentes deben ser adecuados, aunque están limitados por la percepción de ayudas públicas, ofreciéndose a cambio beneficios intangibles como la experiencia y prestigio.

Un marco regulatorio adecuado que garantice la máxima transparencia en las operaciones y procesos también es un elemento importante. El "banco malo" debe tener un estatus legal especial. Aunque no llega a ser necesaria una licencia bancaria como tal, éste debe tener libre acceso a los mercados de capitales y a la financiación del banco central, y aligeradas las cargas administrativas del marco regulatorio y legal de las entidades financieras. Por último, y no por ello menos importante, es la relación con los todos "stakeholders" (accionistas, trabajadores, reguladores, agencias de rating, inversores y el público en general). De especial importancia son las relaciones que se mantengan con agencias de rating e inversores para poder conseguir financiación privada y con los acreedores para que mantengan la calma y confíen. En este sentido, la política de comunicación es clave (Aggarwal et al., 2012).

En el caso concreto de la crisis del sector bancario español, se creó a finales de 2012 la Sociedad de Gestión de Activos Procedentes de la Reestructuración Bancaria, S.A., más conocida por su acrónimo: Sareb. Esta sociedad está dentro de la categoría de "banco malo" con gestión centralizada, pero con capacidad limitada a los activos problemáticos de vida infinita y finita, procedentes de las entidades de crédito sometidas al proceso de reestructuración. 


\section{Conclusiones}

Una vez expuestas las características y modo de funcionamiento del "banco malo" y comprobando que en experiencias pasadas se ha configurado como un mecanismo efectivo de saneamiento para sectores bancarios en crisis, se llega a la conclusión que se hace necesario informar a la ciudadanía de una forma más sencilla y clara sobre lo que son los "bancos malos" de tal forma que no lleve a equívocos. Así, se ha de considerar que la acepción que se da a este mecanismo, "banco malo", no es acertada por dos motivos.

Uno, el más significativo, porque su calificación de "malo" da a entender que no es adecuado ni positivo. Esa acepción viene dada porque a él llegan los activos "malos" de las entidades financieras en crisis. Por el contrario, este instrumento es un vehículo que permite dar salida a unos activos problemáticos que estaban presentes en las entidades financieras con problemas, no sólo ya de liquidez, sino incluso de solvencia. El traspaso de esos activos problemáticos hacia los "bancos malos" supone el saneamiento financiero de estas entidades financieras, deshaciéndose de esos activos que le ahogaban financieramente. Y la segunda razón se basa en que el "banco malo" no es un banco, sino una sociedad (anónima en el caso del Sareb) creada con un objetivo muy concreto con el respaldo financiero del Estado. Transferidos esos activos "malos" de las distintitas entidades financieras con problemas financieros a una sociedad específica, el negocio de esta sociedad es exclusivamente dar salida al mercado a esos activos tóxicos. El establecimiento de una sociedad específica, con expertos especialmente elegidos para esa tarea y con un consejo de administración con una estructura diversificada sienta las bases para una gestión profesionalizada y rigurosa para conseguir el objetivo de dar salida a los activos problemáticos en el mercado en el plazo objetivo dado.

Por tanto, considerando las necesidades de información a la ciudadanía en un ámbito tan técnico como es el financiero, se hace necesario comunicar de una forma más clara y sencilla, haciendo llegar un mensaje tranquilizador de que las autoridades competentes toman medidas para dar soluciones a los problemas y retos que plantea una crisis. En este sentido, cabe destacar los esfuerzos que el Sareb está realizando en comunicación para que la ciudadanía al autodefinirse como una sociedad con naturaleza compleja señalando concretamente las siguientes cuestiones:

"No es un banco, no cuenta con licencia bancaria ni tiene vocación de financiar a los inversores minoristas. No es la mayor inmobiliaria de Europa, su cartera está compuesta en un $80 \%$ por activos financieros y la vivienda es una parte menor de los activos. No tiene hipotecas de particulares, excluidas del perímetro de activos transferidos a Sareb. No es una agencia pública de vivienda social, el objetivo único de Sareb es desinvertir. No participa del Fondo Social de Viviendas creado para atender el problema de los desahucios. No tiene vocación de crecimiento y permanencia, su objetivo es liquidar su cartera" (Sareb, 2013). 


\section{Referencias bibliográficas}

ACHARYA, V. y MATTHEW, R. (2009). Restoring Financial Stability: How to Repair a Failed System. New York: Wiley Finance.

AGGARWAL, S., ARIMOTO, K., BRENNA, G., CLARK, J., GUSE, F. y HÄRLE, P. (2012): "Good riddance. Excellence in managing wind-down portfolios". En McKinsey Working Papers on Risk, $\mathrm{n}^{\mathrm{o}} 31$. April.

BERGSTROM, C.; ENGLUND, P. y THORELL, P. (2003): "Securum and the Way out of the Swedish Banking Crisis". Summary of a report commissioned by SNS - Center for Business and Policy Studies, May.

BIS (2008): 78th Annual Report. Basel, Switzerland.

BIS. (2009): 79th Annual Report. Basel, Switzerland.

BORIO, C. y NELSON, W. (2008): "Monetary operations and the financial turmoil". En BIS Quarterly Review. March, pp. 31-46.

BORIO, C., VALE, B. y PETER, G. (2010): "Resolving the financial crisis: are we heeding the lessons form the Nordics". En BIS Working Papers, $\mathrm{n}^{\circ} 311$. June.

CALOMIRIS, C.W. (2008): "The Subprime Turmoil: What's Old, What's New, and What's Next". En Maintaining Stability in a Changing Financial System, 21-23.

CLAESSENS, S.; DJANKOV, S. y KLINGEBIEL, D. (1999): "Financial Restructuring in East Asia: Halfway There?". En Financial Sector Discussion Paper, ${ }^{\circ}$ 3. The World Bank.

COMISIÓN EUROPEA (2009): Comunicación de la Comisión sobre el tratamiento de los activos cuyo valor ha sufrido un deterioro en el sector bancario comunitario (2009/C 72/01). Diario Oficial de la Unión Europea de 26 de marzo de 2009.

DIAMOND, D.W. y RAJAN, R. (2009): "The Credit Crisis: Conjectures about Causes and Remedies". En American Economic Review, n 99, pp. 606-610.

EICHENGREEN, B.; MODY, A., NEDELJKOVIC, M. y SARNO, L.(2009): "How the Subprime Crisis Went Global: Evidence from Bank Credit Default Swap Spreads". En NBER Working Paper n 14904 (April).

ERGUNGOR, O. (2007): "On the Resolution of Financial Crises: The Swedish Experience". En Federal Reserve Bank of Cleveland, Policy Discussion Papers.

FREIXAS, X; PARIGI, B.M. y ROCHET, J.C. (2003): "The Lender of Last Resort: A 21 st Century Approach”. En Working Paper Series, n 298, European Central Bank.

GROSSMAN, E. y WOLL, C. (2012):"Saving the Banks: The Political Economy of Bailouts". En Sciences Po Paris.

HARVEY, C.R. (2008): "The Financial Crisis of 2008: What Needs to Happen after TARP". En Working Paper, Duke University.

KLINGEBIEL, D. (2000): "The Use of Asset Management Companies in the Resolution of Banking Crises: Cross-country Experiences". En The World Bank, Policy Research Working Paper Series, $\mathrm{n}^{\circ} 2284$.

KROSZNER, R. S.; LAEVEN, L. y KLINGEBIEL, D. (2007): "Banking crises, financial dependence, and growth". En Journal of Financial Economics, vol. 84, $\mathrm{n}^{\mathrm{o}} 1$, pp. 187-228. 
LANG, L.; POULSEN, A. y STULZ, R. (1995): “Asset sales, firm performance, and the agency costs of managerial discretion". En Journal of Financial Economics, vol. $37, \mathrm{n}^{\mathrm{o}} 1$, pp. 3-37.

LAEVEN, L. y VALENCIA, F. (2008):"Systemic banking crises: a new database". En IMF Working. Paper WP/08/224.

LAEVEN, L. y VALENCIA, F. (2010): "Resolution of Banking Crises: The Good the Bad and the Ugly". En International Monetary Fund. Vol. 10

LAEVEN, L. y VALENCIA, F. (2012): "Systemic banking crises database: An update". En IMF Working Paper/12/163, June.

PRAET, P.; NGUYEN, G. (2008): "Overview of Recent Policy Initiatives in Response to the Crisis". En Journal of Financial Stability, no 4, pp. 368-375.

SAREB (2013): "La reforma del Sistema Financiero", IV Encuentro, 9 de julio de 2013. Disponible en: http://www.sareb.es/cms/estatico/srb/sareb/web/es/portal/prensa/ presentaciones/presentaciones/Jornadas_expansion9jul2013.pdf [20-07-2013]

SCHÄFER, D. y ZIMMERMANN, K.F. (2009): "Bad Bank(s) and the Recapitalisation of the Banking Sector". En Intereconomics, July/August, pp. 215-225.

SHLEIFER, A. y VISHNY, R.W. (2012): "Liquidation Values and Debt Capacity: A Market Equilibrium Approach". En The Journal of Finance vol. 47, issue 4, pp. 1343-1366.

SWAGEL, P. (2009): "The financial crisis: An inside view". En Brookings Papers on Economic Activity.

TAYLOR, J.B. (2009): "The Financial Crisis and the Policy Responses: An Empirical Analysis of What Went Wrong”. En NBER Working Paper No. 14631.

\section{La autora}

$\mathrm{M}^{\mathrm{a}}$ del Carmen de la Orden de la Cruz. Actualmente, Profesora Titular (interina) de la Universidad Rey Juan Carlos de Madrid en el Departamento de Economía de la Empresa. Doctora en Ciencias Económicas y Empresariales y Máster en Gestión Financiera por la Universidad Complutense de Madrid. Desde 2007, Personal Docente e Investigador de la Universidad Rey Juan Carlos de Madrid en el Departamento de Economía de la Empresa, donde desarrolla actividades docentes, de gestión (lanzamiento y Directora del Máster Universitario en Asesoramiento y Planificación Financiera de la URJC entre 2009 y 2012) y de investigación. Anteriormente, entre 1990 y 1997, gestora de fondos de inversión en Gesmadrid, S.G.I.I.C. del grupo Caja Madrid y, entre 1998 y 2007, economista del Servicio de Estudios de Caja Madrid (actual Bankia). 